\title{
Contribution à l'étude de l'évolution chimique et du traitement des saumures de fromagerie
}

\author{
par \\ J. L. LACRAMPE, J. HARDY, J. P. RAMET \\ et F. WEBER \\ Ecole Supérieure de Laiterie de Nancy
}

\section{I. - INTRODUCTION}

De nombreuses études ont été effectuées sur les saumures de fromagerie en vue d'améliorer leur qualité et de prolonger leur durée d'utilisation, mais elles n'ont porté que sur leur composition et sur les types de sels à utiliser.

C'est ainsi que Schwarz [23] a étudié en 1940 la composition des bains de sel destinés à la fromagerie. Schulz [22] en 1951, a publié un article sur la composition des saumures. Schragi [21] en 1952 a étudié la composition idéale de bains de sel pour un fromage de type Pinzgau. En 1964, Zivkovic [25] a suivi l'évolution des saumures destinées au salage de fromage blanc et en particulier les teneurs en azote total et en azote soluble.

Différentes méthodes de contrôle des bains de sel ont été proposées en 1962 par Kurmann [11] et en 1964 par Roward [20]. Par ailleurs, on trouve dans la littérature divers articles portant sur la qualité des sels à utiliser. Magakyan [14] en 1957, a étudié un type de sel particulier et son influence sur la qualité des fabrications. Popov [18] a préconisé l'addition d'acide sorbique à la saumure pour empêcher le développement de divers micro-organismes à la surface des fromages. Citons également, dans cet ordre d'idées, les travaux effectués à l'Ecole Supérieure de Laiterie de Nancy par Masson [16] et Mendez [17] sur des sels spéciaux additionnés de phosphate monocalcique et d'acide citrique.

Cependant très peu d'études ont porté sur l'évolution chimique des saumures bien que ce problème préoccupe depuis longtemps le technicien. Nous avons donc dans notre travail abordé ce sujet. 
Dans un premier temps notre recherche a eu pour but de déterminer les causes de ce que l'on appelle généralement le « vieillissement des saumures ", phénomène lié à l'augmentation de leurs charges en impuretés consécutive à leur utilisation répétée. Pour cela, nous avons suivi l'évolution de différents facteurs chimiques dans une saumure destinée au salage de fromages à pâte molle du type Camembert.

Dans un deuxième temps nous avons appliqué à ces saumures divers traitements physico-chimiques afin de tenter de prolonger leur utilisation. Les travaux relatifs à ces traitements sont peu nombreux.

C'est ainsi que Futschik [6] en 1961 a proposé une stérilisation du bain de saumure au moyen d'hypochlorite de sodium ; cette méthode n'apporte qu'une amélioration d'ordre microbiologique. Dans le même but deux autres traitements furent appliqués :

- en 1964 par Hinterberger [9] : utilisation d'eau oxygénée,

- en 1968 par Casalis, Luquet et Rossier [3] : action des rayons ultraviolets.

Viard [24] en 1965, décrit également un procédé de destruction des microorganismes indésirables susceptibles d'affecter la qualité des fromages. En ce qui nous concerne, en vue d'améliorer la qualité chimique et bactériologique d'une saumure «vieillie », nous avons appliqué deux traitements consécutifs : tout d'abord une neutralisation puis une stérilisation. La neutralisation a été réalisée de façon classique par addition de soude et la stérilisation par traitetement thermique.

\section{II. - MATERIELS - METHODES}

Les essais que nous avons effectués ont été réalisés à la fois sur des saumures expérimentales et sur des saumures industrielles.

L'étude expérimentale était destinée à suivre de façon précise l'évolution d'une saumure par passages réguliers de quantités contrôlées de fromages. Par contre celle des saumures industrielles nous a donné la possibilité de juger des variations physico-chimiques d'une saumure utilisée dans les conditions moins rigoureuses de l'usine.

De façon à pouvoir comparer plus précisément les résultats, les lots de fromages saumurés ont été fabriqués dans les mêmes conditions.

\section{a) Fabrication des Camemberts}

La fabrication a été réalisée de façon industrielle par le procédé Corblin sur un lait de grand mélange, dans des conditions d'emprésurage, de coagulation et d'égouttage classiques. 


\section{b) Saumurage des fromages}

\section{- Préparation des bains}

Dans les deux cas les saumures ont été préparées à partir de sels spéciaux* de composition suivante :

$\begin{array}{lll}\mathrm{H}_{2} \mathrm{O} \text { p. } 100 & : & 0,025 \\ \text { Matières insolubles } & : & 0 \\ \text { Sulfates p. } 100\left(\mathrm{SO}_{4}^{--}\right) & : & 0,012 \\ \mathrm{Ca} \text { p. } 100 & : & \text { traces } \\ \mathrm{Mg} \text { p. } 100 & : & 0 \\ \mathrm{~K} \text { p. } 100 & : & 0\end{array}$

La concentration initiale des bains en chlorures a été fixée à $265 \mathrm{~g} / \mathrm{l}$ de solution.

\section{- Conditions d'utilisation des bains et échantillonnage}

Pour les deux catégories de saumures le temps de saumurage a été de $35 \mathrm{mn}$ et la température de $14^{\circ} \mathrm{C}$. Les saumures industrielles ont été rechargées en sel tous les jours en fonction d'un contrôle de densité ; les prises d'échantillons ont été effectuées tous les matins avant passage des différents lots de fromages. L'importance de ces lots était de 960 Camemberts pour $1600 \mathrm{l}$ de saumure.

Les saumures expérimentales ont été utilisées dans des conditions légèrement différentes ; en particulier nous n'avons procédé à aucune recharge en sel de façon à ne pas faire varier les concentrations relatives des différents composants du bain en raison de son faible volume.

Avant prélèvement de la saumure en vue des analyses celle-ci a été préalablement débarrassée des morceaux de caillé par filtration sur un tamis métallique grossier.

Une deuxième filtration a été effectuée ultérieurement sur l'échantillon de façon à éliminer les fines particules de caséine subsistantes.

Les saumures industrielles ne subissaient aucun traitement de filtration ou de tamisage. Par contre les échantillons prélevés étaient toujours filtrés au laboratoire.

Il faut remarquer, à propos de ces saumures industrielles, qu'il y a eu parfois trois passages de fabrications en $24 \mathrm{~h}$ ce qui nous a amenés à définir un " coefficient de charge " des bains comme nous le verrons plus loin.

* Sel fin super épuré, non traité, aimablement fourni par la Compagnie des Salins du Midi et des Salines de l'Est. 


\section{c) Analyses}

Tout au long des essais nous avons contrôlé parallèlement les saumures expérimentales et les saumures industrielles.

En dehors des contrôles courants sur les fabrications ellesmêmes (Matière grasse du lait de mélange, acidité, $\mathrm{pH}$, mesure du

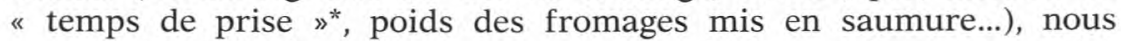
avons effectué diverses analyses sur les bains après passage des différents lots de fromages :

- Poids de la saumure - Densité - Teneur en chlorures - Azote total - Azote non protéique - Acidité Dornic - pH - Extrait sec Teneur en lactose - Teneur en $\mathrm{Ca}$ - Teneur en $\mathrm{Mg}$.

1) Dosage des chlorures

Le taux des chlorures dans les saumures étant très élevé, la méthode Charpentier-Volhard ne nous a pas donné suffisamment de précision pour évaluer la perte en chlorures dans la solution.

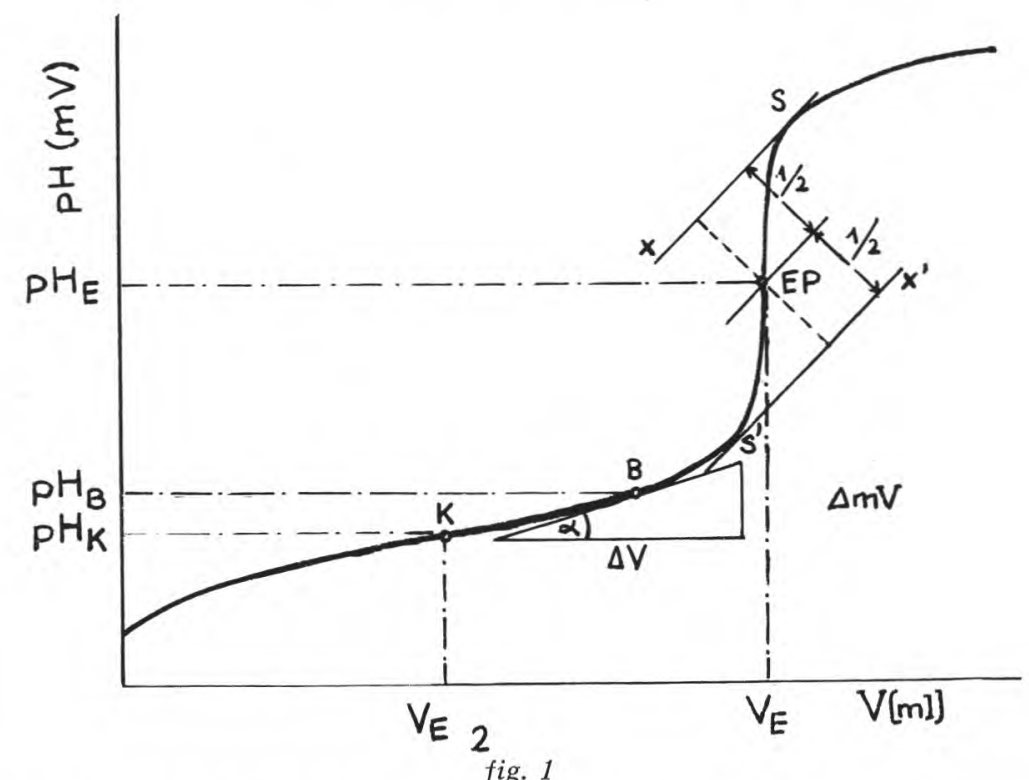

Courbe de variation de la différence de potentiel enregistrée en fonction de l'addition de volumes croissants (V) de $\mathrm{Ag} \mathrm{NO}_{3}, 0,1 \mathrm{~N}$

Ep. : point d'inflexion atteint pour un volume $\mathrm{V}_{\mathrm{E}}$ de nitrate d'argent et déterminé par l'intersection de la parallèle équidistante aux deux tangentes parallèles SX S'x' et de la courbe de titration.

* Nous entendons par " temps de prise " la période qui sépare l'emprésurage du début de la floculation. 
Nous avons donc appliqué une méthode de dosage potentiométrique par combititreur. Le principe de ce dosage est le suivant:

Entre une électrode d'argent, une électrode au $\mathrm{KCl}$ et une solution $\mathrm{Cl}^{-}$, s'établit une différence de potentiel que l'on peut lire sur l'échelle en mv d'un pHmètre. Quand on neutralise les ions $\mathrm{Cl}^{-}$par titrage avec des ions $\mathrm{Ag}^{+}$, la d.d.p. diminue. Au moment où les derniers ions $\mathrm{Cl}^{-}$sont neutralisés, la d.d.p. subit une très forte diminution et en choisissant des concentrations adéquates des réactifs on peut enregistrer ces variations sur les courbes de titration. Le point final de la réaction est caractérisé (fig. 1) par le point d'inflexion de la courbe. Il peut être déterminé de plusieurs façons :

- Détermination graphique (fig. 1) : on trace 2 tangentes parallèles et on prend le milieu de la distance de ces 2 parallèles.

- Méthode par observation des bonds de l'aiguille : on détermine le point d'inflexion en observant la variation de potentiel produite par une faible addition de réactif. La goutte produisant la variation maximum du potentiel est la goutte permettant de situer la fin de la réaction.

Avec l'appareil que nous avons utilisé* il est possible d'afficher le point où l'on veut arrêter le dosage et un générateur d'impulsions conduit le titrage jusqu'à ce point.

Les dosages ont été réalisés à l'aide de solutions de nitrate d'argent $0,1 \mathrm{~N}$ sur des prélèvements de $1 \mathrm{~cm}^{3}$ de saumure additionnés de $100 \mathrm{~cm}^{3}$ d'eau et de $2 \mathrm{~cm}^{3}$ d'acide nitrique concentré.

\section{2) Dosage de l'azote}

Le dosage des différentes fractions azotées a été réalisé par la technique micro-Kjeldahl ; la minéralisation effectuée sur $2 \mathrm{ml}$ de saumure à l'aide de $3 \mathrm{ml}$ d'acide sulfurique concentré. Après refroidissement du matras il s'est avéré nécessaire de diluer le contenu avant transvasement avec de l'eau distillée, celui-ci étant solidifié en raison de la forte teneur en sel. Pour doser l'azote non protéique, la précipitation des protéines a été faite au moyen d'acide trichloracétique à 24 p. 100 ; le précipité a été séparé ensuite par centrifugation pendant $10 \mathrm{mn}$. La minéralisation a porté sur $2 \mathrm{ml}$ de surnageant.

\section{3) Dosage des minéraux : calcium - magnésium}

Ces dosages ont été effectués par spectrophotométrie de flamme. Afin de tenir compte de la présence d'éléments interférents, nous avons employé la méthode dite de "l'étalon interne ». Cette méthode consiste à mesurer les densités optiques des saumures

\footnotetext{
* Combititreur Metrohm type 3 D.
} 


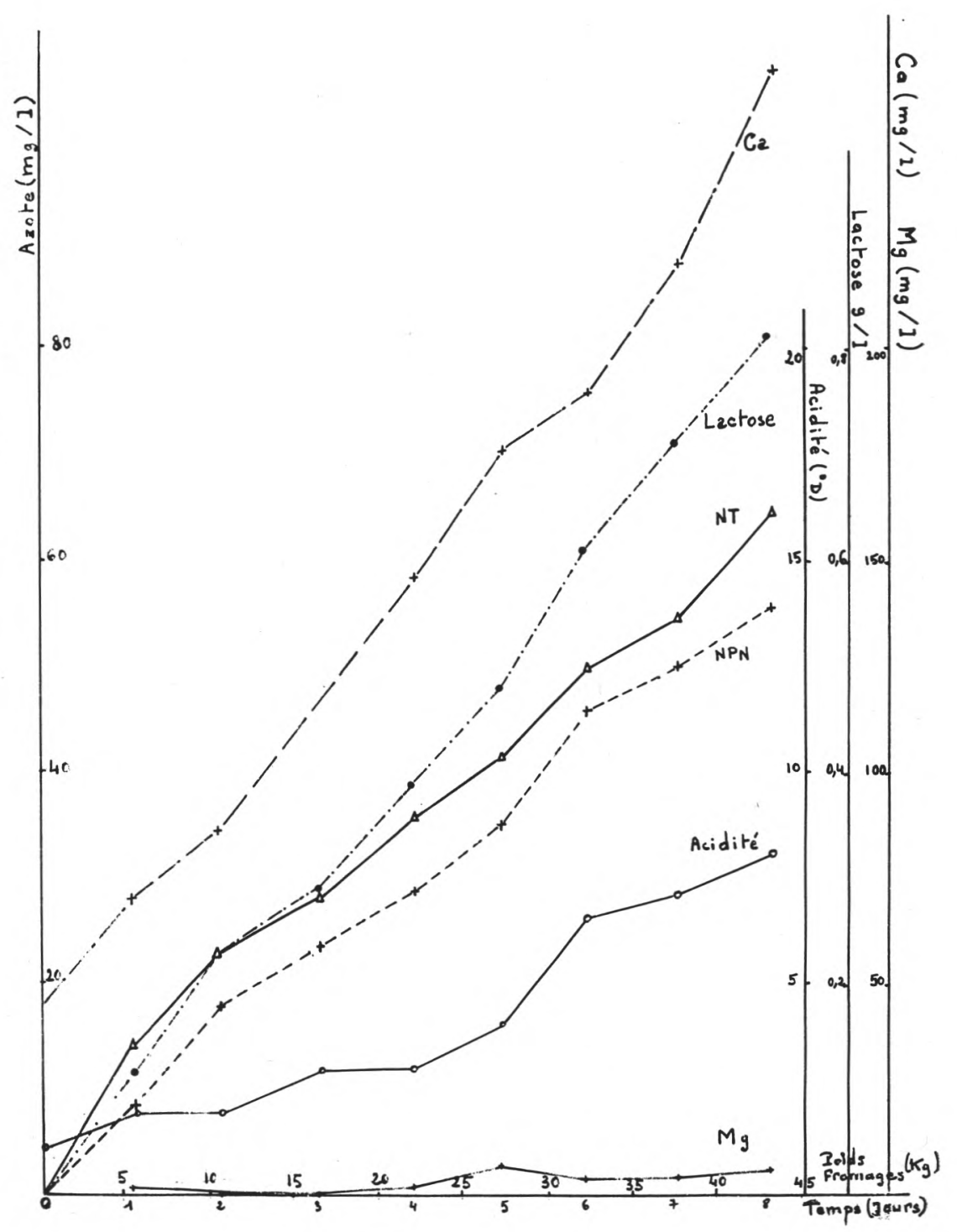

fig. 2

Variation de la composition chimique d'une saumure expérimentale en fonction de la durée d'utilisation 
(après dilution en raison de leurs fortes teneurs en $\mathrm{NaCl}$ ) avec ou sans addition de l'élément à doser. De cette façon les conditions physico-chimiques sont les mêmes dans tous les cas.

\section{4) Dosage du lactose}

Nous avons utilisé pour ce dosage une méthode de polarimétrie électronique. Les échantillons de saumure ont été stabilisés par l'adjonction d'une goutte de formol afin d'éviter la transformation de lactose en acide lactique.

Les autres mesures ont été effectuées par les méthodes classiques ; elles n'ont pas posé de problèmes particuliers.

\section{d) Expression des résultats}

L'évolution des deux types de saumures a été suivie pendant $10 \mathrm{j}$ consécutifs après passage journalier de quantités connues de fromages. Vu la disproportion existant entre les volumes des bains expérimentaux $(201)$ et industriels (1600 1) nous avons été amenés à définir un " coefficient de charge " correspondant au rapport : poids de fromages saumurés

poids de saumure

lière " et une charge totale.

La « charge journalière » correspond au coefficient évalué pour le poids de fromages salés chaque jour, la « charge totale » à celui calculé pour le poids de fromages saumurés au cours de toute la période d'utilisation du bain de sel.

Ainsi, dans nos essais, le volume de la saumure industrielle a été de 16001 soit : $1840 \mathrm{~kg}$ et le poids de fromages journalier de $576 \mathrm{~kg}$ en moyenne. D'où le coefficient de "charge journalière » : 0,31 . Le " coefficient de charge " totale pour $10 \mathrm{j}$ d'utilisation s'est élevé à :

$$
\frac{5472}{1840}=2,98
$$

Pour les saumures expérimentales ces coefficients ont été respectivement : 0,22 et 1,86 .

\section{III. - RESULTATS}

\section{a) Acidité $\mathrm{pH}$}

Dans toutes les saumures que nous avons étudiées, la solution salée de départ a une acidité voisine de $1^{\circ} \mathrm{D}$. Cette acidité augmente ensuite de façon régulière (fig. 2 et 3 ) pour atteindre après 10 saumurages 8 à $10^{\circ} \mathrm{D}$ dans les saumures expérimentales et 25 à 29० D dans les saumures industrielles. 


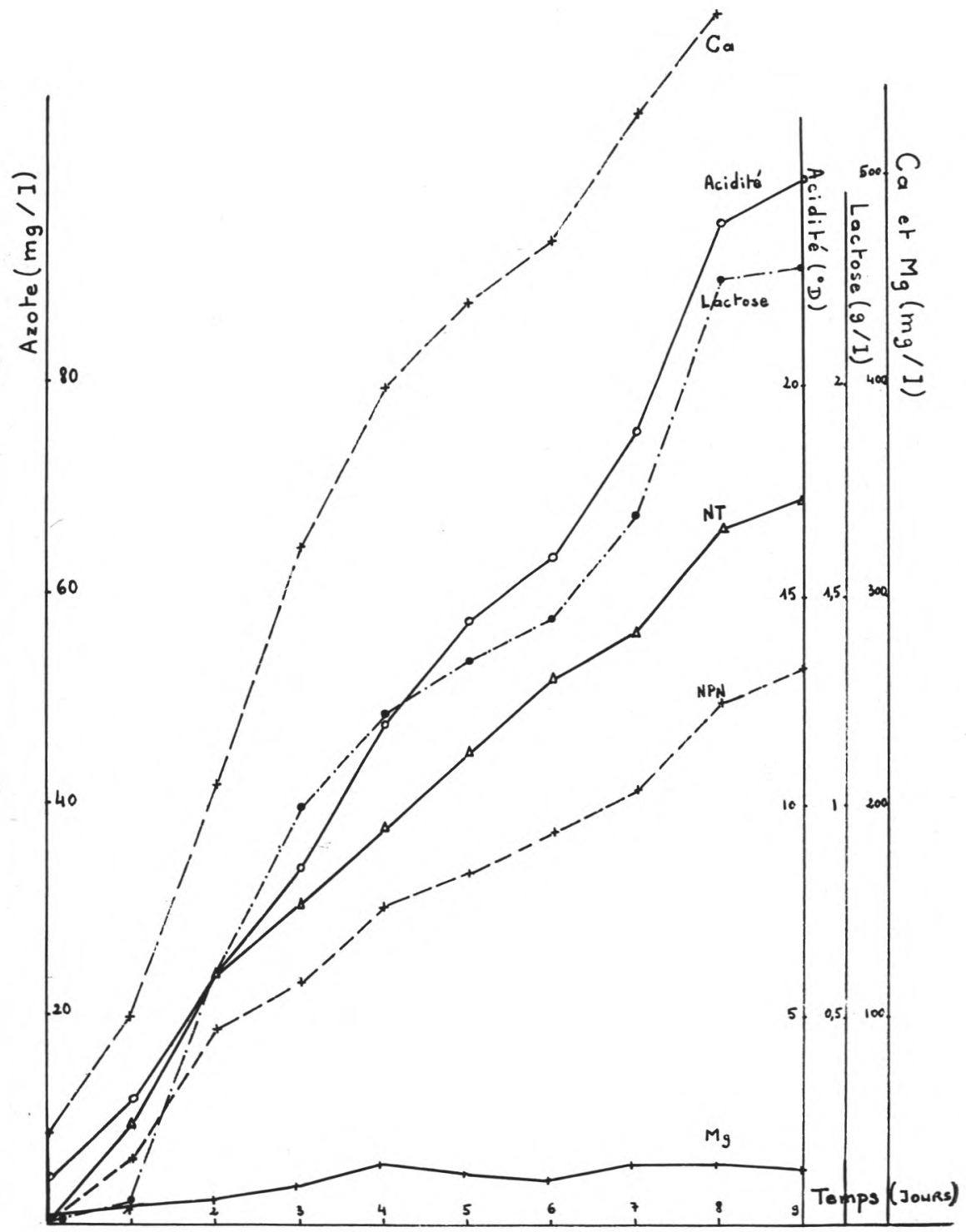

fig. 3

Variation de la composition chimique d'une saumure industrielle en fonction de la durée d'utilisation 
TABLEAU 1. - Résultats analytiques relatifs à l'évolution de la composition chimique d'une saumure expérimentale

\begin{tabular}{|c|c|c|c|c|c|c|c|c|c|}
\hline Temps (jours) & 1 & 2 & 3 & 4 & 5 & 6 & 7 & 8 & 9 \\
\hline Extrait sec $(\mathrm{g} / \mathrm{l}) \ldots \ldots$ & 265,38 & 260,95 & 258,41 & 256,92 & 255,46 & 248,21 & 244,50 & 242,63 & 238,51 \\
\hline Densité $\ldots \ldots \ldots \ldots \ldots$ & 1,170 & 1,165 & 1,165 & 1,165 & 1,160 & 1,160 & 1,155 & 1,155 & 1,150 \\
\hline Acidité $\left({ }^{\circ} \mathrm{D}\right) \quad \ldots$ & 1 & 2 & 2 & 3 & 3 & 4 & 6,5 & 7 & 8 \\
\hline $\begin{array}{lllllllll}\mathrm{pH} & . . & \ldots & \ldots & \ldots & . . & \ldots & . .\end{array}$ & 6,25 & 6,15 & 6,05 & 5,95 & 5,80 & 5,60 & 5,35 & 5,10 & 4,80 \\
\hline Chlorures $(\mathrm{g} / \mathrm{l})$. & 265,2 & 260,1 & 256,6 & 253,6 & 249,4 & 243,6 & 239,4 & 236,5 & 231,9 \\
\hline Lactose $(\mathrm{g} / \mathrm{l}) \ldots \ldots \ldots$ & 0 & 0,12 & 0,23 & 0,29 & 0,39 & 0,48 & 0,61 & 0,71 & 0,81 \\
\hline Azote total $(\mathrm{mg} / \mathrm{l}) \ldots \ldots$ & 0 & 14 & 22,6 & 28 & 35,7 & 41,4 & 49,8 & 54,4 & 64,4 \\
\hline $\mathrm{NPN}(\mathrm{mg} / \mathrm{l}) \quad \ldots$ & 0 & 8,4 & 18,2 & 23,8 & 28,7 & 35 & 45,5 & 49,7 & 55,3 \\
\hline Calcium $(\mathrm{mg} / \mathrm{l}) \quad \ldots \quad \ldots \quad .$. & 45 & 70 & 86 & 116 & 146 & 176 & 190 & 220 & 266 \\
\hline Magnésium $(\mathrm{mg} / \mathrm{l}) \ldots \ldots$ & 0 & 2 & 0 & 0 & 2 & 7 & 4 & 4 & 6 \\
\hline Poids des fromages $(\mathrm{kg})$ & 0 & 5,5 & 10,84 & 16,48 & 22,08 & 27,24 & 32,28 & 37,80 & 43,30 \\
\hline
\end{tabular}


TABLEAU 2. - Résultats analytiques relatifs à l'évolution de la composition chimique d'une saumure industrielle

\begin{tabular}{|c|c|c|c|c|c|c|c|c|c|c|}
\hline Temps (jours) & 1 & 2 & 3 & 4 & 5 & 6 & 7 & 8 & 9 & 10 \\
\hline Extrait $\sec (\mathrm{g} / \mathrm{l}) \ldots \ldots \ldots$ & 270,75 & 255,61 & 253,64 & 266,0 & 271,85 & 279,36 & 274,38 & 275,14 & 269,50 & 270,20 \\
\hline Densité $\ldots \begin{array}{llllll} & \ldots & \ldots & \ldots & \ldots & \ldots\end{array}$ & 1,180 & 1,170 & 1,170 & 1,170 & 1,175 & 1,180 & 1,175 & 1,175 & 1,170 & 1,170 \\
\hline Acidité $\left({ }^{\circ} \mathrm{D}\right) \quad \ldots \ldots c c c$ & 1 & 3 & 6 & 8,5 & 12 & 14,5 & 16 & 19 & 24 & 25 \\
\hline $\begin{array}{lllllllll}\mathrm{pH} & \ldots & \ldots & \ldots & . & . . & . & .\end{array}$ & 7,2 & 6,25 & 5,3 & 5,25 & 5,05 & 4,95 & 4,90 & 4,80 & 4,70 & 4,70 \\
\hline Chlorures $(g / 1) \quad \ldots \quad \ldots .$. & 270,56 & 253,15 & 248,8 & 262,95 & 266,90 & 273,78 & 258,27 & 268,36 & 257,40 & 258,13 \\
\hline Lactose $(\mathrm{g} / \mathrm{l}) \ldots \ldots \ldots$ & 0 & 0,06 & 0,61 & 1 & 1,22 & 1,35 & 1,45 & 1,70 & 2,26 & 2,29 \\
\hline Azote total $(\mathrm{mg} / \mathrm{l}) \ldots$ & 0 & 24,35 & 59,5 & 77 & 95,2 & 113,4 & 131,6 & 142,1 & 167,65 & 172,9 \\
\hline 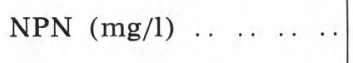 & 0 & 16,1 & 46,9 & 58,1 & 76,3 & 84,4 & 93,8 & 104,3 & 125,3 & 133,7 \\
\hline Calcium $(\mathrm{mg} / \mathrm{l}) \ldots \ldots$ & 44 & 100 & 210 & 324 & 400 & 440 & 470 & 530 & 580 & 620 \\
\hline Magnésium $(\mathrm{mg} / \mathrm{l}) \ldots$ & 5 & 10 & 12 & 18 & 29 & 24 & 20 & 28 & 28 & 25 \\
\hline
\end{tabular}


Cette augmentation d'acidité est due, d'une part à l'acide lactique exsudant du fromage et d'autre part au lactose se transformant en acide lactique sous l'influence des bactéries lactiques dans la saumure.

Quant au $\mathrm{pH}$ de départ, il semble être lié à la composition du sel, mais surtout au $\mathrm{pH}$ de l'eau servant à préparer les saumures ; pour le même sel, nous avons en effet, obtenu 7,1 puis 6,30.

En fin d'utilisation des saumures les $\mathrm{pH}$ atteints ont été de 4,55 et 4,70 pour les saumures industrielles et de 4,80 à 5,95 pour les saumures expérimentales (fig. 2 et 3 ).

\section{TABLEAU 3}

Résultats comparatifs de l'évolution de la densité et du taux de chlorures

\begin{tabular}{|c|c|c|c|c|c|}
\hline Densité & \multicolumn{3}{|c|}{$\begin{array}{l}\text { Valeurs mesurées } \\
\quad(\mathrm{g} / \mathrm{de} \mathrm{NaCl})\end{array}$} & $\begin{array}{l}\text { Moyenne } \\
(\mathrm{g} / \mathrm{l})\end{array}$ & $\begin{array}{c}\text { Incertitude } \\
\text { moyenne }\end{array}$ \\
\hline 1,130 & $\begin{array}{l}191,3 \\
193,6\end{array}$ & & & 192,4 & 1,15 \\
\hline 1,135 & $\begin{array}{l}196,2 \\
199,4 \quad(\end{array}$ & $\begin{array}{c}202,7 \\
(211,7)\end{array}$ & & 199,4 & 2,1 \\
\hline 1,140 & $\begin{array}{l}205,6 \\
208,8\end{array}$ & 214,7 & & 209,7 & 3,3 \\
\hline 1,150 & $\begin{array}{l}213,9 \\
230,1\end{array}$ & & & 231,0 & 0,9 \\
\hline 1,155 & $\begin{array}{l}236,5 \\
237,2\end{array}$ & $\begin{array}{l}239,4 \\
239,6\end{array}$ & 244,1 & 239,4 & 2,0 \\
\hline 1,160 & $\begin{array}{l}243,6 \\
245,7 \\
249,4\end{array}$ & $\begin{array}{l}249,5 \\
253,5 \\
261,1\end{array}$ & & 250,4 & 4,5 \\
\hline 1,165 & $\begin{array}{c}(237,2) \\
253,6 \\
256,6\end{array}$ & $\begin{array}{l}257,0 \\
260,1 \\
265,2\end{array}$ & & 258,5 & 3,3 \\
\hline 1,170 & $\begin{array}{l}246,5 \\
247,1 \\
248,8\end{array}$ & $\begin{array}{l}252,1 \\
253,1 \\
257,4\end{array}$ & $\begin{array}{l}258,1 \\
265,2 \\
262,9\end{array}$ & 254,8 & 5,4 \\
\hline 1,175 & $\begin{array}{l}254,6 \\
258,3\end{array}$ & $\begin{array}{l}268,4 \\
266,9\end{array}$ & & 262,0 & 5,6 \\
\hline 1,180 & $\begin{array}{l}273,8 \\
270,6\end{array}$ & $\begin{array}{l}270,7 \\
269,3\end{array}$ & & 271,1 & 1,1 \\
\hline
\end{tabular}


Par ailleurs, on remarque une chute très rapide du $\mathrm{pH}$ en début d'utilisation de la saumure qui s'explique par l'apport important d'acide lactique.

\section{b) Chlorures}

Dans le cas des saumures expérimentales qui n'étaient pas " rechargées » en sel, on constate une décroissance très régulière du taux de chlorures après passage des fabrications. La perte en sel de la saumure a été évaluée à $0,79 \mathrm{~g} / \mathrm{l}$ par $\mathrm{kg}$ de fromage immergé, soit 1,58 p. 100 de la teneur initiale.

Ce résultat est tout à fait comparable aux chlorures dosés dans les fromages puisque nous avons trouvé dans ces derniers un taux de 1,63 p. 100 de $\mathrm{NaCl}$.

Dans le cas des saumures industrielles, il est difficile de tirer des conclusions étant donné qu'elles ont été " rechargées " en sel quotidiennement en tenant compte uniquement de la densité. Les taux de chlorures obtenus sont très variables. Ceci montre que l'on commet de graves erreurs en " rechargeant " les saumures en se basant uniquement sur la densité (tab. 3).

\section{c) Densité}

Nous avons constaté que, pour une même densité, le taux de chlorures trouvé dans la saumure pouvait être très variable.

Aussi nous avons regroupé les résultats obtenus dans un tableau (tab. 3), et calculé la moyenne des résultats ainsi que la moyenne des écarts à la moyenne. Nous voyons de cette façon que la mesure de la densité ne donne une idée du contenu en chlorures de la saumure qu'à $\pm 5 \mathrm{~g} / 1$ près environ.

\section{d) Extrait sec}

La variation de l'extrait $\mathrm{sec}$ est tout à fait identique à celle des chlorures (fig. 2 et 3). Cependant, la différence entre taux d'extrait sec et taux de chlorures croît avec la durée d'utilisation de la saumure ; elle correspond aux impuretés contenues dans la solution salée.

Nous obtenons en fin d'utilisation des taux d'impuretés de $12,1 \mathrm{~g} / \mathrm{l}$ et $15,7 \mathrm{~g} / 1$ pour les deux saumures industrielles et des taux de $6,6 \mathrm{~g} / 1$ et 9,3 g/l pour les saumures expérimentales. Ces résultats sont tout à fait comparables compte tenu des coefficients de " charge totale » différents.

\section{e) Taux d'azote}

\section{1) Azote total}

Nous constatons une augmentation régulière de la teneur en azote total dans les saumures, qu'elles soient industrielles ou expérimentales (fig 2 et 3 ). Après 10 j d'utilisation, le taux d'azote total 
varie entre $57 \mathrm{mg} / \mathrm{l}-69 \mathrm{mg} / \mathrm{l}$ pour les saumures expérimentales et $172,9 \mathrm{mg} / 1$ - $196 \mathrm{mg} / 1$ pour les saumures industrielles. On relève dans ce dernier cas, même en tenant compte des coefficients de charge, une quantité plus importante d'azote qui peut s'expliquer par l'absence de filtration.

\section{2) Azote non protéique}

Les taux d'azote non protéique sont très voisins de ceux de l'azote total (fig. 2 et 3 ). La variation du taux de NPN se fait ici encore de façon croissante pour les deux types de saumures. L'écart constaté a semble-t-il, les mêmes causes que ci-dessus.

\section{3) Taux de lactose}

La teneur en lactose augmente parallèlement à l'acidité (fig. 2 et 3) dans le cas des saumures industrielles comme dans celui des saumures expérimentales. Il est vraisemblable que ce phénomène est le résultat d'un échange osmotique entre la solution et le fromage.

4) Minéraux $(\mathrm{Ca}, \mathrm{Mg})$

La teneur en calcium subit une augmentation de $200 \mathrm{mg} / \mathrm{l}$ pour les saumures expérimentales et de $600 \mathrm{mg} / 1$ pour les saumures industrielles en $10 \mathrm{j}$ d'utilisation (fig. 1 et 2 ).

La proportion de magnésium a toujours été très faible quelles que soient les saumures.

Il apparaît donc à la lumière des résultats obtenus, que le "vieillissement " de la saumure correspond en fait à une augmentation du taux des impuretés. Nous avons résumé dans un tableau les taux des différents éléments dosés après $10 \mathrm{j}$ d'utilisation des saumures et nous avons exprimé tous les résultats en fonction d'un coefficient de "charge totale" ramené à 1 (tab. 4). Ceci nous permet de mieux comparer l'évolution des saumures expérimentales et des saumures industrielles.

\section{Traitement des saumures}

Après avoir étudié l'évolution des saumures, nous avons pensé qu'il serait intéressant d'abaisser les taux d'impuretés par des traitements appropriés, ce qui aurait pour conséquence de prolonger leur utilisation et d'améliorer leur qualité.

\section{1) Méthode de traitement}

Sur des saumures chargées en impuretés nous avons appliqué en laboratoire un traitement combiné de neutralisation et de chauffage. Nous avons procédé selon le protocole suivant :

a) Filtration sur papier.

b) Neutralisation par $\mathrm{NaOH} \mathrm{N}$.

c) Stérilisation par chauffage à $110^{\circ} \mathrm{C}$ pendant $20 \mathrm{mn}$.

d) Filtration sur papier. 


\section{TABLEAU 4}

Variations des taux de différents constituants chimiques rapportés à un coefficient de charge totale égal à 1 dans des saumures industrielles et expérimentales après $10 \mathrm{j}$ d'utilisation

\begin{tabular}{|c|c|c|c|c|c|}
\hline & $\begin{array}{c}\text { Saumure } \\
\text { expérimentale } \\
1\end{array}$ & $\begin{array}{c}\text { Saumure } \\
\text { expérimentale } \\
2\end{array}$ & $\begin{array}{l}\text { Saumure } \\
\text { expérimentale } \\
3\end{array}$ & $\begin{array}{c}\text { Saumure } \\
\text { industrielle } \\
1\end{array}$ & $\begin{array}{c}\text { Saumure } \\
\text { industrielle } \\
2\end{array}$ \\
\hline 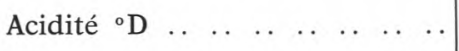 & 5,3 & 4,3 & 4,5 & 8,3 & 9,3 \\
\hline $\mathrm{pH}$ & 0,62 & 0,77 & 0,69 & 0,83 & 0,88 \\
\hline E.S. - Chlorures $(\mathrm{g} / \mathrm{l}) \ldots \ldots \ldots$ & 2,8 & 3,55 & 5,0 & 4,02 & 5,2 \\
\hline 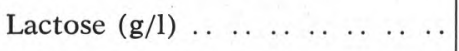 & 0,40 & 0,43 & 0,42 & 0,76 & 0,90 \\
\hline Azote total $(\mathrm{mg} / \mathrm{l}) \ldots \ldots c c c c$ & 28,2 & 30,8 & 33,5 & 54,8 & 63,0 \\
\hline 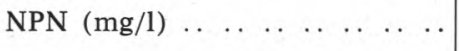 & 20,9 & 25,9 & 27,1 & 41,4 & 44,1 \\
\hline 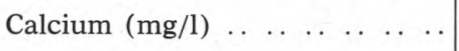 & 107,5 & 119 & 119 & 192 & 206 \\
\hline Magnésium $(\mathrm{mg} / \mathrm{l}) \ldots \ldots \ldots c c c$ & 4,5 & 3 & 1 & 6,6 & 6,3 \\
\hline
\end{tabular}




\section{2) Résultats}

Nos résultats portent sur 6 saumures industrielles après $10 \mathrm{j}$ d'utilisation.

Nous avons analysé ces saumures avant et après traitement ; nous avons par ailleurs pesé le précipité obtenu après neutralisation et chauffage (tab, 5). Outre l'acidité ramenée à $0^{\circ} \mathrm{D}$, nous constatons une diminution de 20 p. 100 du taux de calcium. La proportion d'azote total subit une baisse de 30 p. 100 alors que le taux de NPN diminue de 10 p. 100 seulement. Le traitement semble donc éliminer une partie des protéines.

Ce mode de traitement nous a paru intéressant à divers titres:

- le point de vue bactériologique est évident,

- la diminution de l'acidité a pour intérêt de faciliter les échanges osmotiques en saumure (qui sont favorisés par une acidité inférieure à $20^{\circ} \mathrm{D}$ ). De plus le développement des levures peut être partiellement inhibé par cette baisse de l'acidité,

- l'élimination d'une partie de l'azote ralentit le développement des germes putréfiants,

- la baisse du taux de calcium diminue les risques de goût amer dans le fromage et augmente la solubilité du sel.

\section{IV. - CONCLUSION}

Il résulte de nos observations que la notion de vieillissement des saumures correspond à une augmentation de leurs taux d'impuretés. Celles-ci se chargent à la suite des passages successifs de fabrications fromagères, de différents éléments tels que : acide lactique, matières azotées, sels minéraux. Ces diverses impuretés sont apportées par les fromages, l'eau et le sel. Lorsque leurs concentrations s'élèvent, elles peuvent être à l'origine directe de défauts du fromage comme par exemple l'apparition de goûts indésirables. Indirectement, elles constituent un milieu de culture favorable au développement de microorganismes variés qui contribuent à accélérer le processus de "vieillissement ".

La neutralisation puis la stérilisation de saumures usagées provoquent une diminution sensible des taux des contaminants notamment du calcium, des matières azotées et de l'acide lactique.

Pourtant l'application de ces procédés à l'échelon industriel ne peut guère être envisagée en raison de leurs coûts élevés. Il n'est pas possible en effet, d'employer dans le cas présent des appareils en acier inoxydable ordinaire en raison des effets de corrosion très importants provoqués par le passage des saumures concentrées et chaudes ; l'emploi d'aciers spéciaux au titane et au titane-palladium s'avère indispensable et nécessite un investissement très important. Dans ces conditions, le traitement préconisé, qui en définitive, compte 
TABLEAU 5

Effets du traitement combiné, neutralisation, stérilisation, sur la composition de saumures industrielles

\begin{tabular}{|c|c|c|c|c|c|c|}
\hline & $\mathrm{A}_{1}$ & $\mathbf{B}_{1}$ & $\mathrm{C}_{1}$ & $\mathrm{~A}_{2}$ & $\mathrm{~B}_{2}$ & $\mathrm{C}_{2}$ \\
\hline Acidité avant traitement $\left({ }^{\circ} \mathrm{D}\right)$ & 17,5 & 22 & 24 & 20 & 24 & 26 \\
\hline Acidité après traitement $\left({ }^{\circ} \mathrm{D}\right)$ & 0 & 0 & 0 & 0 & 0 & 0 \\
\hline $\begin{array}{l}\text { Azote total avant traitement } \\
(\mathrm{mg} / \mathrm{l}) \ldots . . . \\
\text { Azote total après }\end{array}$ & $\begin{array}{r}142,1 \\
96,2\end{array}$ & $\begin{array}{l}167,6 \\
116,1\end{array}$ & $\begin{array}{l}172,9 \\
123,2\end{array}$ & $\begin{array}{l}158,2 \\
106,6\end{array}$ & $\begin{array}{l}189,7 \\
125,3\end{array}$ & $\begin{array}{l}196 \\
130,5\end{array}$ \\
\hline $\begin{array}{l}\text { NPN avant traitement }(\mathrm{mg} / \mathrm{l}) \\
\text { NPN après traitement }(\mathrm{mg} / \mathrm{l})\end{array}$ & $\begin{array}{r}104,3 \\
92,5\end{array}$ & $\begin{array}{l}125,3 \\
112,6\end{array}$ & $\begin{array}{l}133,7 \\
119,8\end{array}$ & $\begin{array}{l}116,9 \\
103,2\end{array}$ & $\begin{array}{l}126,7 \\
111,9\end{array}$ & $\begin{array}{l}140,7 \\
126,7\end{array}$ \\
\hline $\begin{array}{l}\text { Calcium avant traitement }(\mathrm{mg} / \mathrm{l}) \\
\text { Calcium après traitement }(\mathrm{mg} / \mathrm{l})\end{array}$ & $\begin{array}{r}530 \\
47\end{array}$ & $\begin{array}{r}580 \\
49\end{array}$ & $\begin{array}{r}620 \\
73\end{array}$ & $\begin{array}{r}560 \\
48\end{array}$ & $\begin{array}{r}660 \\
49\end{array}$ & $\begin{array}{r}660 \\
75\end{array}$ \\
\hline $\begin{array}{l}\text { Lactose avant traitement }(\mathrm{g} / \mathrm{l}) \ldots \\
\text { Lactose après traitement }(\mathrm{g} / \mathrm{l}) \ldots\end{array}$ & $\begin{array}{l}1,70 \\
0,84\end{array}$ & $\begin{array}{l}2,26 \\
1,48\end{array}$ & $\begin{array}{l}2,29 \\
1,12\end{array}$ & $\begin{array}{l}2,24 \\
1,81\end{array}$ & $\begin{array}{l}2,50 \\
2,07\end{array}$ & $\begin{array}{l}2,71 \\
1,57\end{array}$ \\
\hline Poids du précipité $(\mathrm{g} / \mathrm{l}) \ldots \ldots$ & 3,11 & 3,60 & 3,55 & 3,32 & 3,58 & 3,18 \\
\hline
\end{tabular}

$A_{1}, B_{1}, C_{1}$ : première série de contrôles.

$A_{2}, B_{2}, C_{2}$ : deuxième série de contrôles. 
tenu des taux d'impuretés subsistants, ne " rajeunit » les bains que de $5 \mathrm{j}$ apparaît difficilement rentable.

\section{Ré s u m é}

L'évolution chimique des saumures de fromagerie consécutive à leur utilisation répétée se caractérise par une augmentation de leurs taux d'impuretés notamment du calcium, de l'acide lactique et des matières azotées. La neutralisation puis la stérilisation de ces saumures "vieillies » permet de diminuer ces taux ; l'application industrielle de ces procédés paraît difficilement réalisable en raison de leurs coûts élevés.

\section{S u $\mathrm{m}$ m a ry}

The chemical evolution of brines in cheese manufacturing after several uses consists in an increase of their impureties especially of lactic acid, calcium and nitrogenous substances. Neutralization and sterilization of used brines reduce these proportions ; the industrial utilisation of these processes seems to be impossible.

\section{Zusammenfassung}

Das chemische Entwicklung der Käsereisalzbäder nach grosser Verwendung zeigt eine Erhöhung der Fremdenkörper wie Kalzium, Milchsaüre, Eiwei $\beta$ stoffe. Durch die Neutralisierung und die Sterilisierung dieser gebrauchten Salzbäder kann man diese Fremdenkörper entfernen ; leider, wegen der hohen kosten dieser Verfahren ist es fast un möglich sie in einen Betrieb zu benutzen.

\section{Bibliographie}

[1] Anderson (O. A.) (1949 b). - Brining of Manor cheese. Mejeritek. Medd., 3,35 .

[2] Bazlov (B.) (1958). - Brining. Mol. Prom., 19, (8), 28.

[3] Casalis (J.), Luquet (F. M.) et Rossier (F.) (1969). - Sur le traitement des saumures de fromagerie par les rayons ultra-violets, Le Lait, 49, p. 134-145.

[4] Centeleghe (J.L) (1970). - Mise au point de techniques d'examens bactériologiques des sels et des saumures de fromagerie. Etudes de survie de quelques souches bactériennes. $61 \mathrm{p}$. dactylgr. (D.E.A. Sciences du Lait, Nancy).

[5] DolezaleK (M.) and Radl (J.) (1959). - Brining and Salt diffusion in Moravian cheese. Prumysl Potravin, 10, 177.

[6] Futschik (J.) (1953). - Salt diffusion in wet and dry salted Tilsit cheese. Int. Dairy Congr., 2, 642.

[7] Futschiк (J.) (1960). - Salt content of semi-hard cheese. Milchwiss, Berichte Wolfpassing., 10, 11. 
[8] Futschiк (J.) $(1961$ c). - Sterilization of brine baths. Ost. Milchw., 16, 233.

[9] Hinterberger (S.) (1964). - Brine and its sterilization. Ost. Milchw., 19, (21), 401-404.

[10] KNez (V.) (1957). - Brine equipment. Prumysl Potravin., 8, 627.

[11] Kurmann (J.) (1962). - Beitrag zur Kenntnis der bakteriellen Verhältnisse in Emmentaler Käsesalzbädern. Path. et Microbiol., 25, 593-609.

[12] Laiterie eXPÉRimentale D'Etat, Hiller $\varnothing$ D (Danemark). - Hurtigmetoder til elektrometrisk saltbestemmelse. Pub., 161.

[13] Linden (G.). - Applications de la spectrophotométrie d'absorption atomique dans les laboratoires d'Industries alimentaires. Ind. Ali. Agric. (à paraître).

[14] Mc Dowal (F. H.) (1938). - Solubility of curd in brine. J. Dairy Res., 9, 339.

[15] MaGaKYAN (1957). - Brine composition and properties of cheese. Trud. Armyan. nauch-issled. Inst. Zhivotn. Vet., 4, 121.

[16] Masson (A.) (1959). - Contribution à l'étude de l'amélioration des sels de fromagerie. Bull. Soc. Sci. Nancy, 28, 1.

[17] Mendez (R.) (1958). - Etude de l'action du sel sur le développement du Penicillium candidum. Bull. Soc. Sci. Nancy, mars, 2.

[18] Popov (K.) (1963). - Kranit. Prom., 11, (4), 36-37.

[19] Posthumus (G.) (1951). - Brining cheese. Off. Org. K. ned. Zuivelb, 43, 612.

[20] Roward. - Méthodes de contrôle des saumures de fromagerie. Station laitière Grangeneuve, Fribourg (Suisse).

[21] Schragi (F.) (1952). - Brine bath for Pinzgau cheese. Der Salzburger Bauer., 7, (20), 9.

[22] Schulz (M. E.) (1951 c). - Brine baths. Süddtsch. Molkereiztg., 71, 819.

[23] Schwarz (G.) (1940). - Brine bath for salting cheese. Molkereiztg., Hildesh., $54,77$.

[24] VIARD (M.) (1965). - Le saumurage en fromagerie. Annuaire des Anciens Elèves de l'Ecole Supérieure de Laiterie de Nancy.

[25] Zivkнovic (1964). - Brine composition. Dairy Sc. Abstr., 1773.

Reçu pour publication en novembre 1970. 